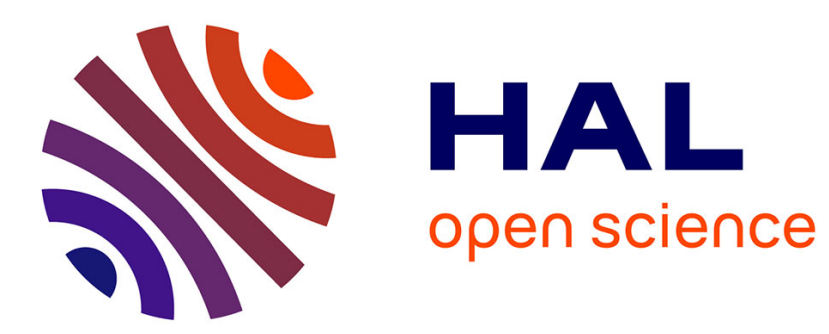

\title{
Abaxial growth and steric constraints guide leaf folding and shape Acer Pseudoplatanus
}

\author{
Etienne E. Couturier, Nicole N. Brunel, Stéphane S. Douady, Naomi N. \\ Nakayama
}

\section{- To cite this version:}

Etienne E. Couturier, Nicole N. Brunel, Stéphane S. Douady, Naomi N. Nakayama. Abaxial growth and steric constraints guide leaf folding and shape Acer Pseudoplatanus. American Journal of Botany, 2012, 99 (8), pp.1289-1299. 10.3732/ajb.1100325 . hal-00964673

\section{HAL Id: hal-00964673 \\ https://hal.science/hal-00964673}

Submitted on 28 May 2020

HAL is a multi-disciplinary open access archive for the deposit and dissemination of scientific research documents, whether they are published or not. The documents may come from teaching and research institutions in France or abroad, or from public or private research centers.
L'archive ouverte pluridisciplinaire HAL, est destinée au dépôt et à la diffusion de documents scientifiques de niveau recherche, publiés ou non, émanant des établissements d'enseignement et de recherche français ou étrangers, des laboratoires publics ou privés. 


\title{
Botany
}

American Journal of Botany 99(8): 1289-1299. 2012.

\section{Abaxial growth and Steric Constraints guide LEAF FOLDING AND SHAPE IN ACER PSEUDOPLATANUS ${ }^{1}$}

\author{
Etienne Couturier ${ }^{2,3}$, Nicole Brunel $^{4,5}$, Stéphane Douady ${ }^{2}$, And NaOmi Nakayama ${ }^{6,7}$ \\ ${ }^{2}$ Laboratoire MSC, UMR 7057 CNRS/Université Paris-Diderot, 10 rue Alice Domon et Léonie Duquet 75205 \\ Paris Cedex 13, France; ${ }^{3}$ Departamento de Física, Universidad de Santiago de Chile, Avenida Ecuador 3493, Estacion Central, \\ Santiago, Chile; ${ }^{4}$ Clermont Université, Université Blaise Pascal, UMR 547 PIAF, F-63000 Clermont-Ferrand, France; \\ ${ }^{5}$ INRA UMR 547 PIAF, F-63000 Clermont-Ferrand, France; and ${ }^{6}$ Institute of Plant Sciences, University of Bern, \\ Altenbergrain 21, Bern CH-3013, Switzerland
}

- Premise of the study: How leaf shape is regulated is a long-standing question in botany. For diverse groups of dicotyledon species, lamina folding along the veins and geometry of the space available for the primordia can explain the palmate leaf morphology. Dubbed the kirigami theory, this hypothesis of fold-dependent leaf shape regulation has remained largely theoretical. Using Acer pseudoplatanus, we investigated the mechanisms behind the two key processes of kirigami leaf development.

- Methods: Cytological examination and quantitative analyses were used to examine the course of the vein-dependent lamina folding. Surgical ablation and tissue culturing were employed to test the effects of physical constraints on primordia growth. The final morphology of leaves growing without steric constraints were predicted mathematically.

- Key results: The cytological examination showed that the lamina's abaxial side along the veins grows substantially more than the adaxial side. The abaxial hypergrowth along the veins and the lamina extension correlated with the lamina folding. When a primordium was released from the physical constraints imposed by the other primordia, it rapidly grew into the newly available space, while maintaining the curvature inward. The morphology of such a leaf was predicted to lack symmetry in the lobe shapes.

- Conclusions: The enhanced growth on the abaxial side of the lamina along the veins is likely to drive lamina folding. The surgical ablation provided clear support for the space-filling nature of leaf growth; thus, steric constraints play a role in determination of the shapes of folded leaves and probably also of the final leaf morphology.

Key words: abaxial growth; Acer pseudoplatanus; Aceraceae; biomechanics; leaf folding; leaf shape determination; maple leaf development; palmate leaf; space-dependent growth; steric constraints.

Leaf morphology is a classic botanical interest. The leaf has become a model system to dissect lateral organ development in plants, and leaf morphogenesis has become a model system to study regulation of plant form (e.g., Sinha, 1999; Fleming, 2005; Braybrook and Kuhlemeier, 2010; Efroni et al., 2010; Hasson et al., 2010; Moon and Hake, 2010). While genetic and physiological studies continue to identify molecular regulators of leaf shapes, contributions of nonmolecular factors, such as the physical constraints acting upon the developing primordia, have recently started to be addressed (Hamant et al., 2008; Prusinkiewicz and Barbier de Reuille, 2010). In particular, transient folding of leaf primordia inside the bud can explain the palmate leaf shapes of a diverse array of dicotyledon species. This observation led to a theoretical characterization of the

${ }^{1}$ Manuscript received 5 July 2011; revision accepted 2 July 2012.

The authors thank Drs. Jacques Dumais (Harvard University, USA), Bruno Moulia (INRA/UMR Piaf, France), and Seisuke Kimura (Kyoto Sangyo University, Japan) for helpful discussion and suggestions and JeanLouis Julien (UMR Piaf, France) and Cris Kuhlemeier (University of Bern, Switzerland) for kindly hosting E.C.'s visits. They also thank Meredith Root-Bernstein and Richard Smith for English proofreading. This work was supported by CNRS, University of Paris-Diderot, French Ministry for Education and Research, Fondecyt (Grant 3120105), EU Adventure Grant MechPlant, European Molecular Biology Organization, Roche Research Foundation, and SystemsX.ch.

${ }^{7}$ Author for correspondence (e-mail: naomi.nakayama@ips.unibe.ch)

doi:10.3732/ajb.1100325 lobed leaf shape formation and formulation of the kirigami theory (Couturier et al., 2009).

The theory proposes that this type of leaf morphogenesis works like kirigami, the Japanese craft in which a piece of paper is first folded and then cut. At the shoot apex under the many layers of older primordia, a young primordium grows via cell division and expansion. As the lamina grows, it folds inward along the veins and again outward between two veins, forming folds and antifolds. This process describes the paper folding part of kirigami. The primordium grows until it fills the available space, halting growth when it meets the other primordia within the bud. Such space-filling growth of leaf primordia has been found in all dicotyledon species examined to date and thus seems to be a widely relevant phenomenon (Couturier et al., 2011). Depending on the species, the geometry of the space available for a primordium delimits the lamina growth on a nearly flat plane: this comprises the cutting part of kirigami. For example, in some plants with decussate phyllotaxy, two opposite leaves grow to meet each other in the middle, mutually inhibiting further growth of the facing primordium (Fig. 1A).

Kirigami is a method to create a repetitive and symmetrical outline on a piece of paper. Similarly, the kirigami regulation acts on the outlines of the leaf lamina. A sinus is the indentation between two lobes (Fig. 1B). Each fold and antifold of a young primordium corresponds to the lobe and sinus of the mature leaf, respectively (Fig. 1B, C). The number and position of the lobes coincide with vein pattern, and the sizes of the lobes are determined by the timing of vein formation. We should note 

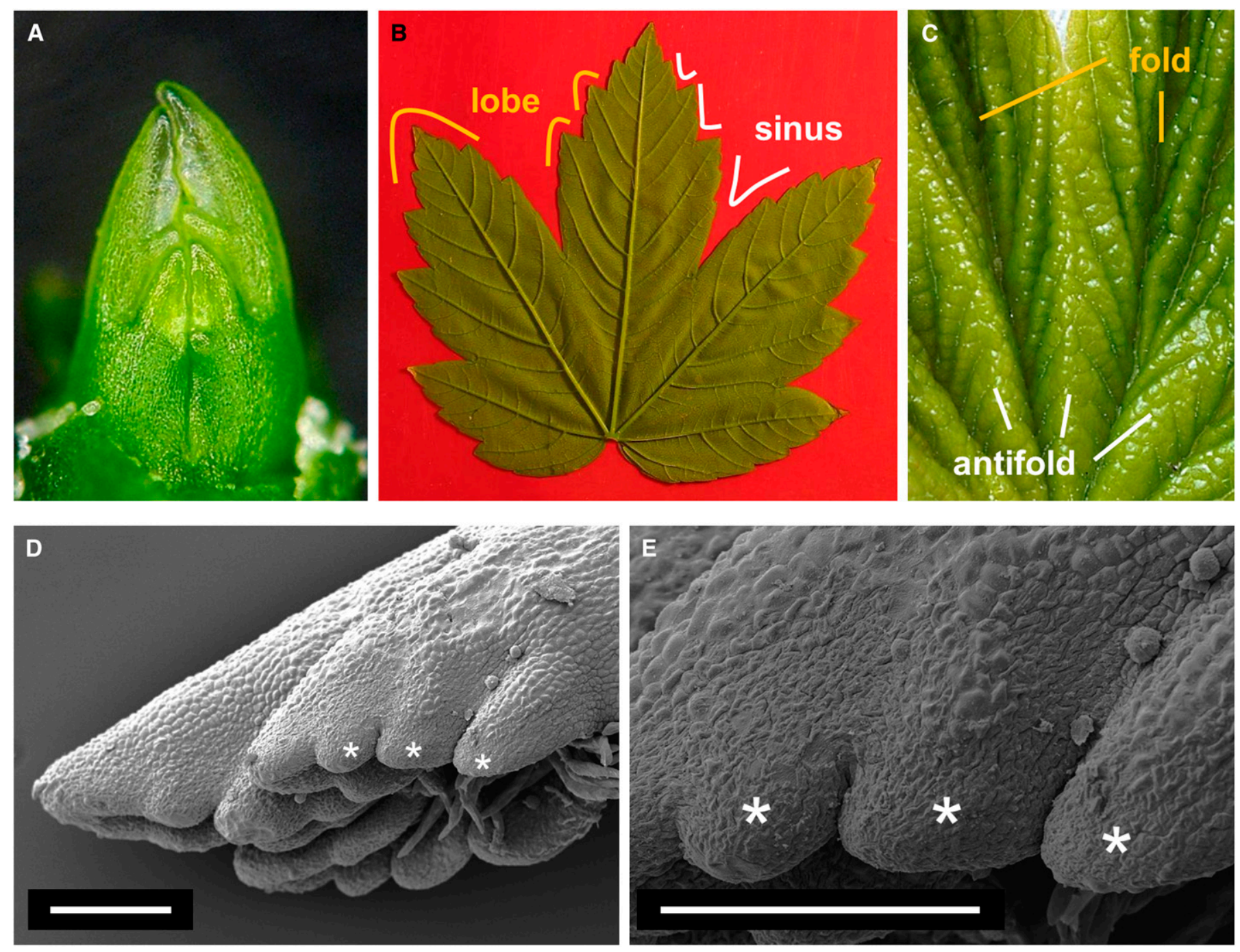

Fig. 1. Morphology and folding of Acer pseudoplatanus leaf. (A) A pair of young leaves. The lamina of the opposite leaves fold and form a near mirror-image set of lobes that press against one another. The lamina margins are positioned along a plane in the middle with fluctuations, indicating reciprocal limitation of growth between the opposite leaves. The lamina folding and mutual inhibition between facing leaves together comprise kirigami leaf morphogenesis and produce the lobed shape of mature, opened leaves. (B) Typical lamina morphology of lobed mature leaf, which correlates with the organization of the developmentally transient folds. The abaxial side of the leaf is shown. (C) Folds and antifolds in an opening leaf, pictured from the adaxial side. (D) Fold formation in a young leaf visualized by scanning electron microscopy. The lamina has already folded along the primary veins, but not yet along the secondary veins. Nicks delimit future small lobes (marked with asterisks) on the lamina margin prior to folding along the secondary veins. Close-up of the lamina before folding (E). Scale bars $=100 \mu \mathrm{m}$.

that we call all tips on the leaf margin lobes, regardless of the more precise terminologies applicable (Fig. 1B). The lobes in a more traditional sense, which we call large lobes, form along the primary veins. The smaller lobes, which are often referred to as serrations or crenations, develop along the secondary and tertiary veins. This generalization stems from the observation that all tips and indentations are indistinguishable in how they form according to the kirigami model. It also is supported by the recent findings that the different degrees of lamina indentations, from leaflet formation to serration, result from a common molecular mechanism (Nikovics et al., 2006; Barkoulas et al., 2008; Blein et al., 2008; Koenig et al., 2009; Kawamura et al., 2010).

The kirigami theory is contingent on two processes of lamina development: folding along the veins and space filling growth. However, the mechanisms underlying transient folding of dicotyledon leaves or contact-dependent growth inhibition are as yet unknown. To gain a greater biological basis for the theory, we have conducted cytological and surgical examinations of kirigami leaf development, using a maple, Acer pseudoplatanus, as an example. The results suggest that a common mechanismasymmetric growth across the abaxial-adaxial axis along the veins - drives the lamina folding and guides the physical constraints acting in the space-dependent growth, emerging as the key process in the kirigami regulation of lobed leaf shape.

\section{MATERIALS AND METHODS}

Plastic sections-Apical buds of Acer peudoplatanus were collected from the campus of les Cezeaux of the University Blaise Pascal in Clermont-Ferrand, France. After dissection to 2-3 mm in length, they were fixed in ice-cold FAA 
A
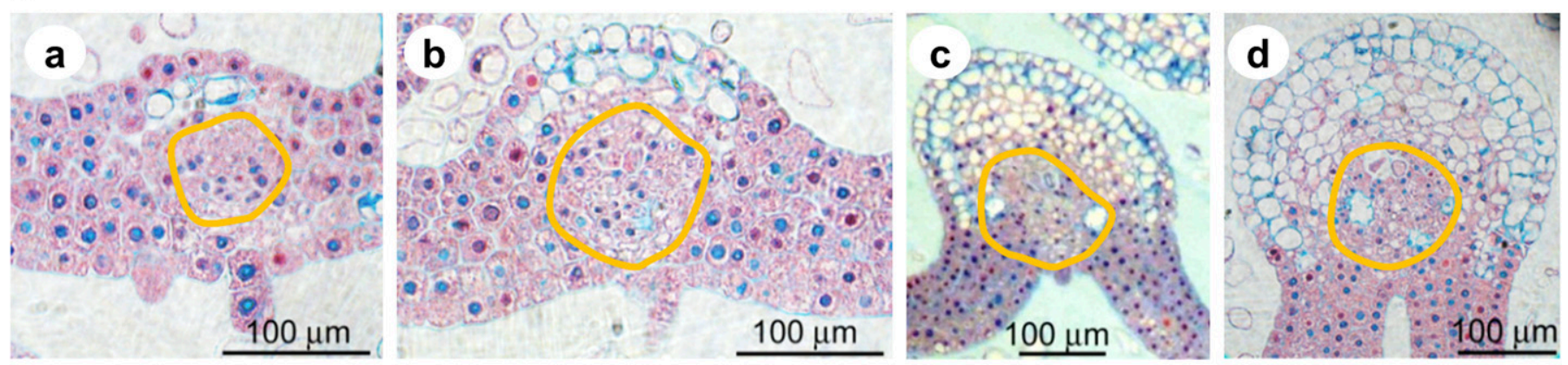

B
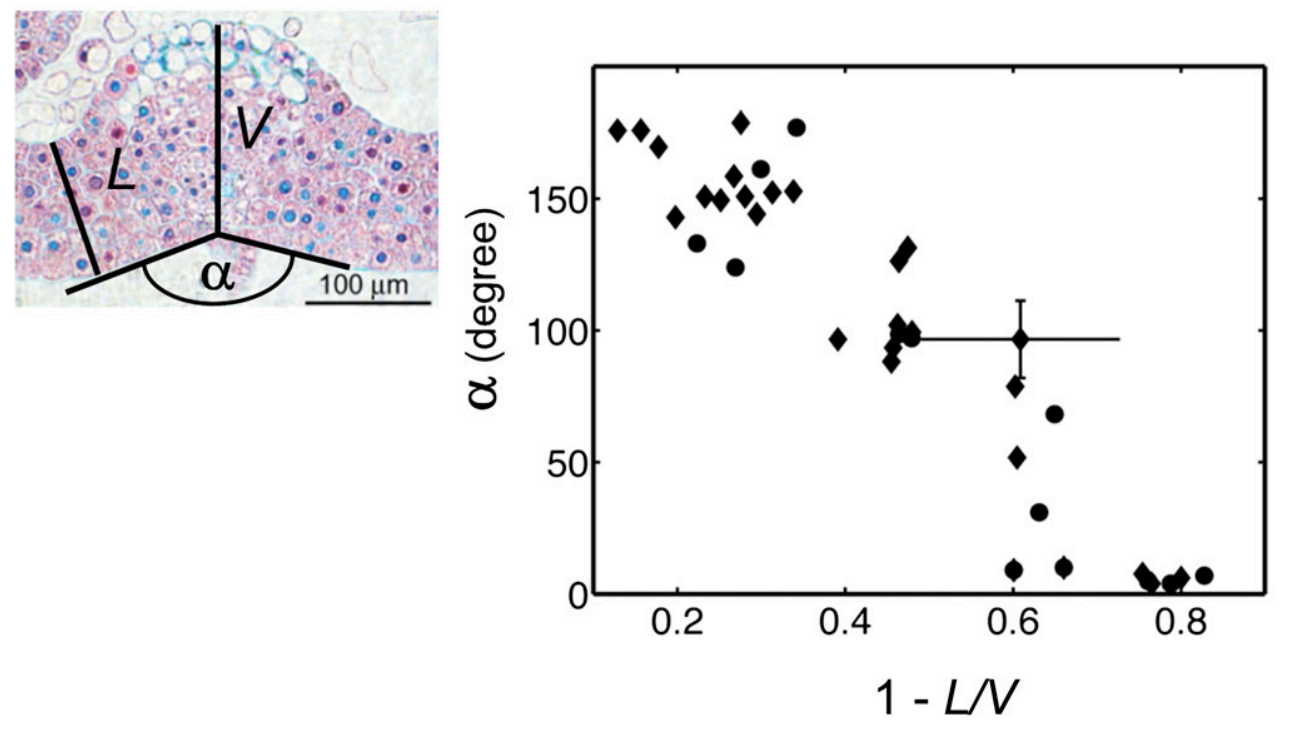

C

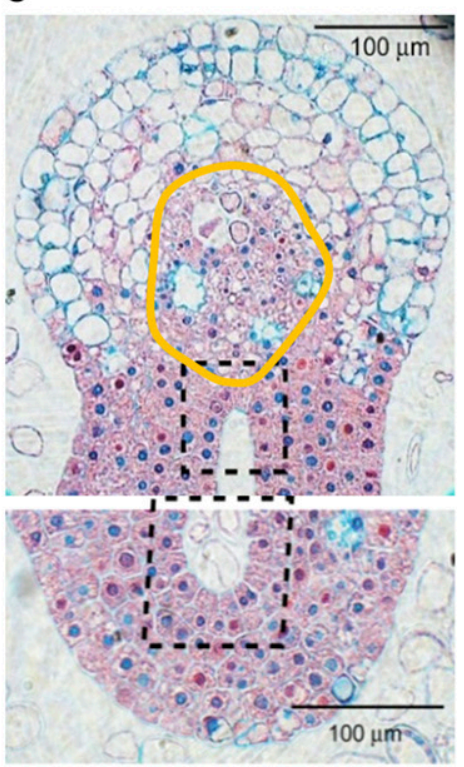

Fig. 2. Correlation between abaxial growth along the vein and fold formation. Because the different orders of veins mature at different times during leaf development, and the size of the veins varies along the proximo-distal axis, we can find a collection of different stages of folding dynamics in one leaf. Transverse sections along the proximo-distal axis were examined. (A) Successive stages (a-d) of vein growth. The cells in the region of the lamina abaxial to the veins grow much more than the adaxial cells. (B) The fold angle follows the vein development. The ratio $L / V$ of the folds normal to the plane of the section was measured from one leaf $(\bullet)$ and its pair leaf $(\bullet)$. Standard deviations are shown as crossed bars. We collected measurements from two opposite leaves within a bud, for which we could obtain every section along the entire leaf. The data collapse on the same curve. (C) Dashed boxes highlight epidermal cells on the adaxial and abaxial sides of the folds and antifolds, respectively. Epidermal cells appear compressed on the adaxial side of the fold and the abaxial side of the antifold, while they appear extensively expanded on the opposite side. Vasculature is marked with an orange line (A, C).

solution (3.7\% formaldehyde, $50 \%$ ethanol, $5 \%$ acetic acid, all v/v), vacuum infiltrated for $1 \mathrm{~h}$ and incubated at $4^{\circ} \mathrm{C}$ for $3-5 \mathrm{~h}$. Tissues were then dehydrated through ethanol series and then gradually embedded into LR White Resin (Sigma Aldrich, St. Louis, Missouri, USA) as reported earlier (Azri et al., 2009). Infiltration was completed in $100 \%$ LR-White resin overnight at $4^{\circ} \mathrm{C}$. The samples were then placed in gelatin embedding capsules, filled with fresh resin, and heat polymerized at $55^{\circ} \mathrm{C}$ for at least $15 \mathrm{~h}$ before semithin $(3-4 \mu \mathrm{m})$ sectioning with a OMU2 microtome (Reichert Technologies, Depew, New York, USA). Semithin sections were stained with Astra blue and safranin. Observations were performed with a light microscope Zeiss Axioplan 2 (Zeiss, Jena, Germany), and data were recorded using a digital camera AxioCam HR with AxioVision digital imaging software (Zeiss).

Surgical ablation experiments-Actively growing axial branches were collected from the Aare river bank in Bern, Switzerland. They were first dissected down to the shoot apex (the meristem and 3-4 pairs of young leaves smaller than $2 \mathrm{~mm}$ ), and some were further dissected for ablation. After dissection, plants were placed onto the growth medium containing $1 / 2 \times$ Murashige-Skoog medium, $1 \%(\mathrm{w} / \mathrm{v})$ sucrose, and $0.5-0.6 \%(\mathrm{w} / \mathrm{v})$ agarose. To prevent infection, Plant Preservative Mixture (Plant Cell Technology, Washington, D.C., USA) was also added to $0.1 \%(\mathrm{v} / \mathrm{v})$. Plants were cultured at $21^{\circ} \mathrm{C}$ and $80 \%$ humidity, in long-day condition ( $16 \mathrm{~h}$ day $/ 8 \mathrm{~h}$ night). Many did not grow well in culture and died within $5 \mathrm{~d}$, while others survived longer than $20 \mathrm{~d}$. Those lasting samples were analyzed $(N=25)$. Growth was recorded daily using Nikon stereoscope SMZ 1500 and Nikon Digital Sight DS-Fil camera with the software NIS-Elements (Nikon Instruments Europe, Amsterdam, Netherlands).

Image analysis-Images were processed and assembled using Adobe Illustrator (San Jose, California, USA) and Microsoft Powerpoint (Redmond, Washington, USA). Noises, such as original scale bars and uneven background signals, were fixed, and the image was processed for similar orientations and the best brightness and contrast.

Data were quantitatively analyzed with the programs Image J (http://rsbweb. nih.gov/ij/) and Matlab 7.7 (MathWorks, Natick, Massachusetts, USA). For Figs. 2 and 3, we used a complete set of transverse plastic sections of two opposite maple leaves (Appendices S1 and S2, see Supplemental Data with online version of article). We analyzed 3-19 successive sections from the mid-height region of the lamina. For Fig. 2, the fold opening angle $\alpha$, the lamina thickness $L$, and the lamina thickness at the vein $V$ were measured for each vein, and the mean was calculated and graphed. The variations in the measurements are shown for a representative vein, as crossed standard deviation lines. For Fig. 3, 
A
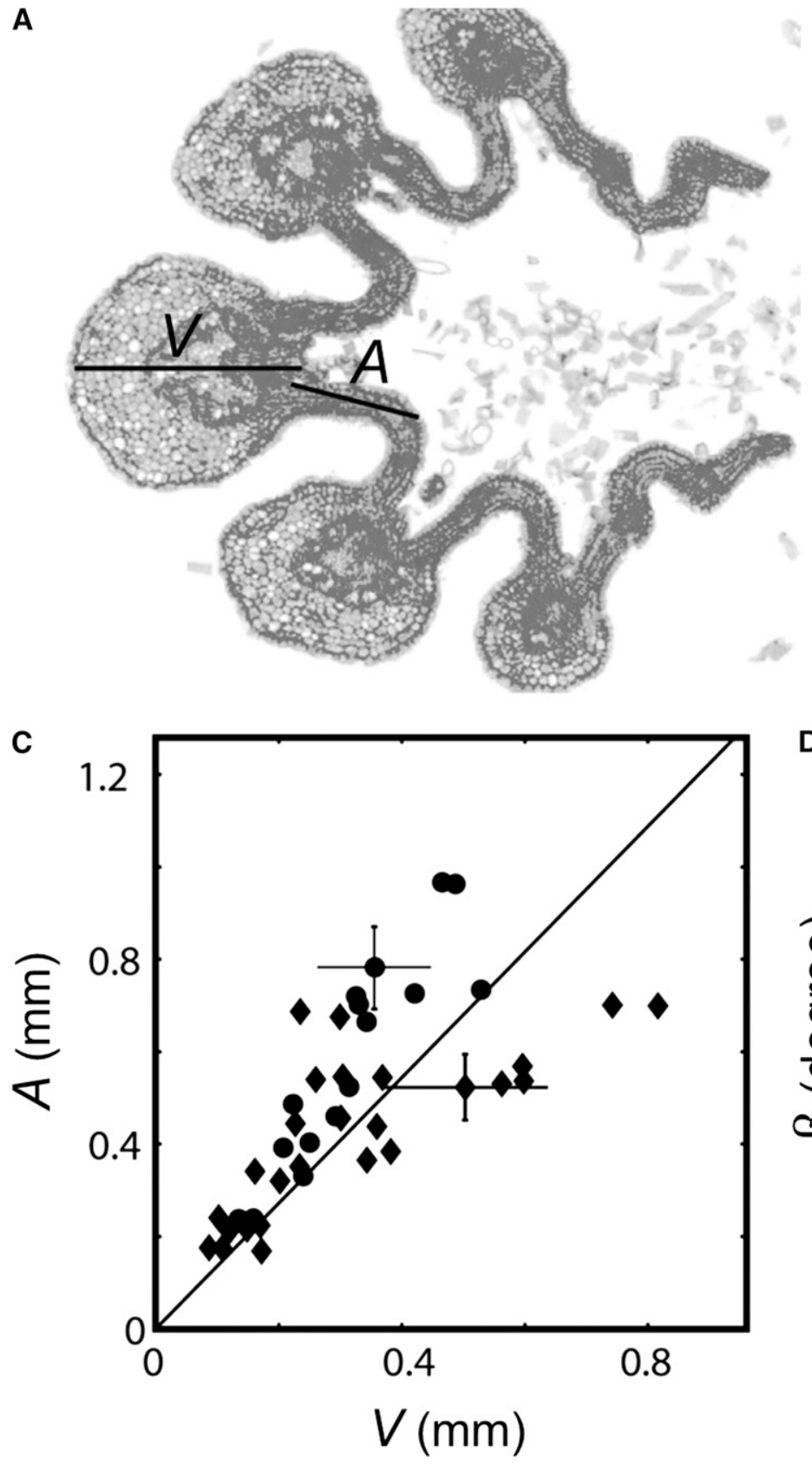
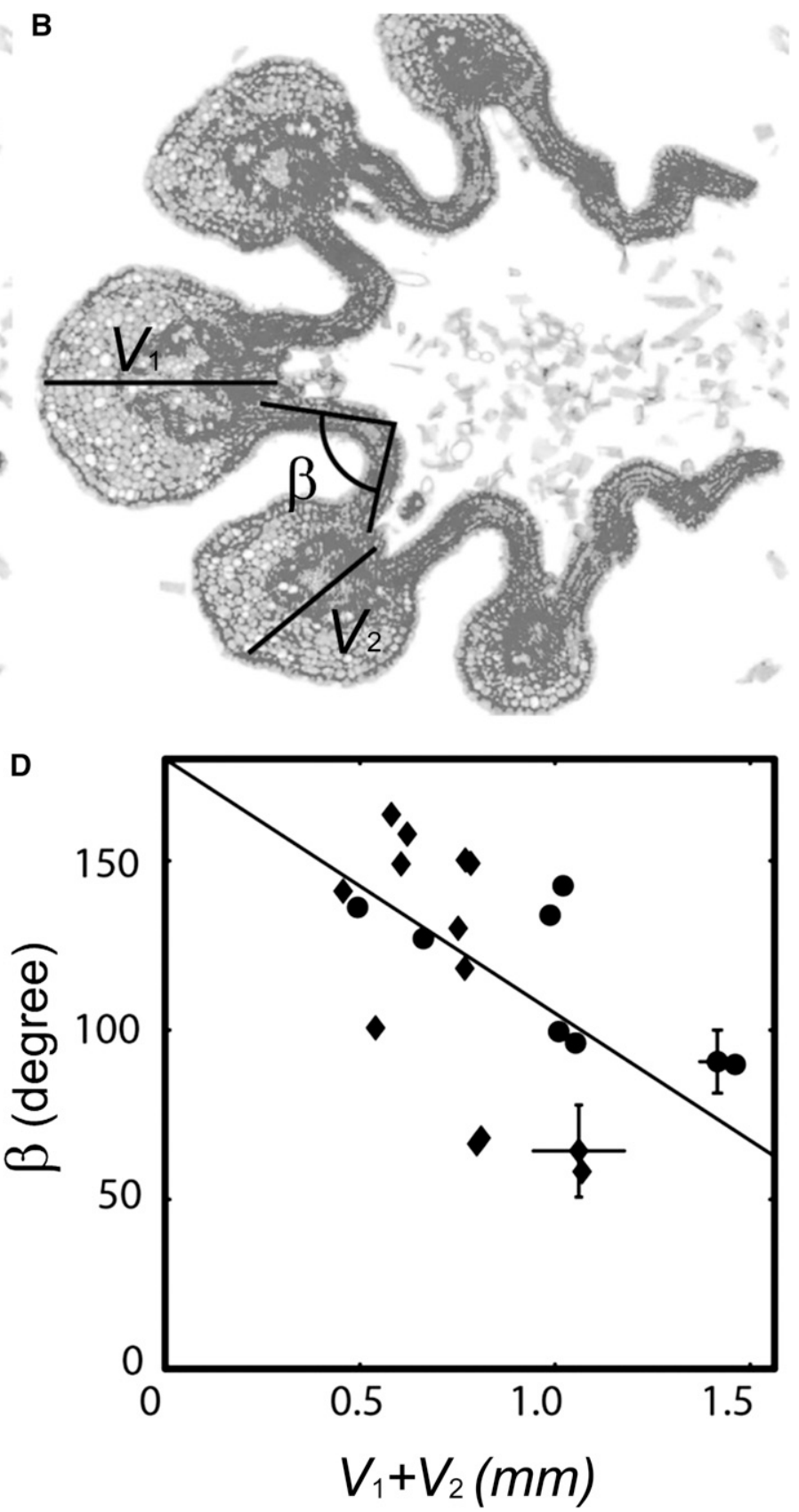

Fig. 3. Antifold formation correlates with lamina growth. The same set of transverse sections of folding leaves as in Fig. 2 were used for examination of antifold formation. (A, C) The lamina thickness along a vein $V$ is plotted as a function of the lamina length $A$ between the vein and the closest antifold. The lamina extension (medio-lateral growth) is proportional to the lamina thickening (abaxial-adaxial growth). Note that most of the lamina thickening is due to abaxial growth. (B, D) The antifold angle $\beta$ is plotted against the sum of the lamina thickness at the two closest veins $V_{1}+V_{2}$. Antifold formation correlates with the abaxial growth along the vein. Each dot represents the mean of 3-19 measurements made for each vein at different proximo-distal positions. $\bullet$ : data from one leaf; $\downarrow$ : data from the pairing leaf. Standard deviation is shown by the crossed error bars for two representative veins.

the distance $A$ between the vein and the antifold (the most curved place) and the lamina thickness at the vein $V$ were measured for each antifold, as well as the antifold angle $\beta$ and the sum of the lamina thickness of the two adjacent veins $V_{1}+V_{2}$. To determine the antifold angle $\beta$, we chose the approximate mid-point of the curved region farthest from the two adjacent veins as the central vertex and where the adaxial side of the lamina meets the two adjacent veins as the lateral vertices. For Fig. 5, the central vein contour was plotted with the Segmented line tool of ImageJ at different time points of growth, and the total turning angle of the vein (the sum of the successive angles between the segments) and the length of the leaf were calculated. For the ablated samples, the remaining leaf was measured, whereas for the control samples both intact leaves were measured. The leaves were monitored every 2 to $3 \mathrm{~d}$ over the $18 \mathrm{~d}$ after the surgery.

\section{RESULTS}

Lamina folding along the vein-At the shoot apex of A. pseudoplatanus, two primordia emerge in decussate phyllotaxy 
A

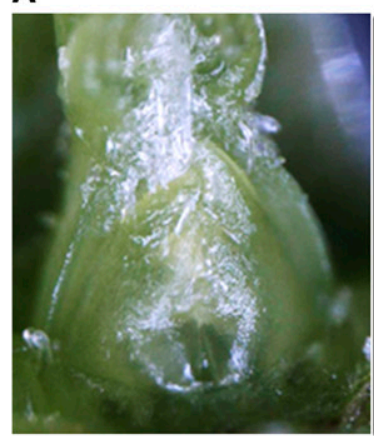

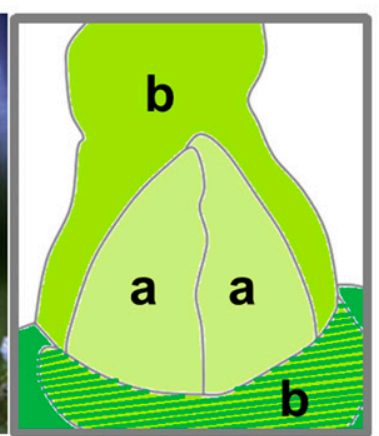

B

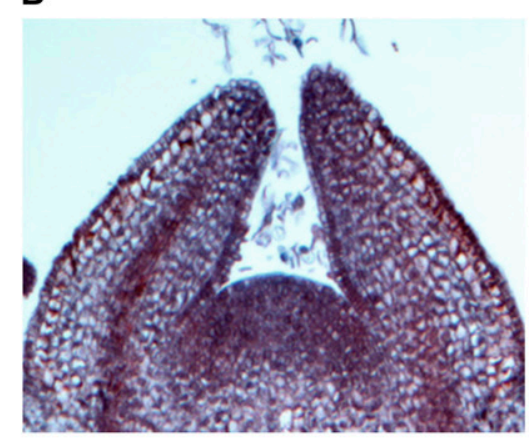

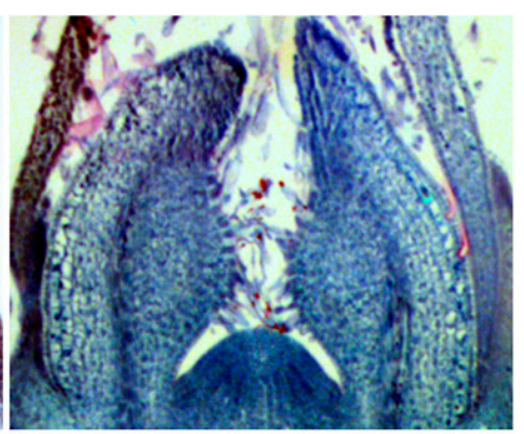

C
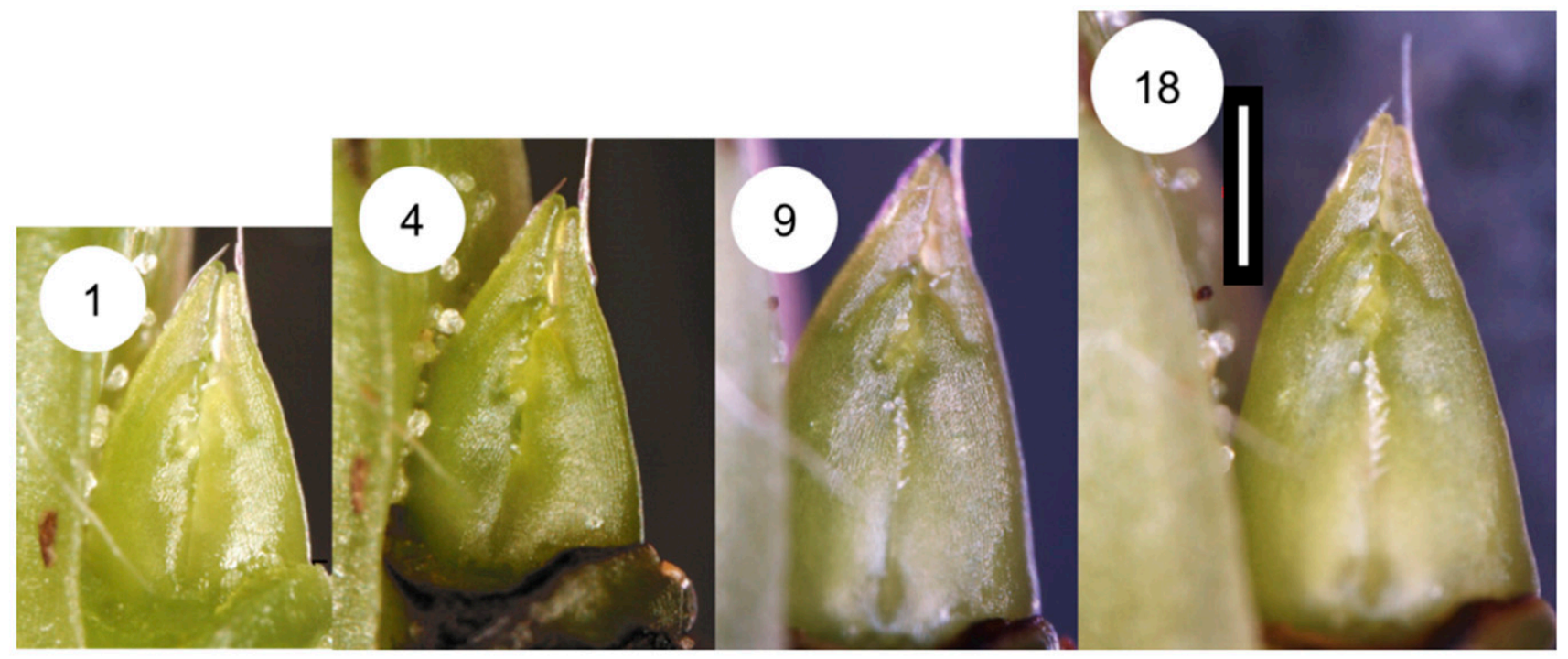

D

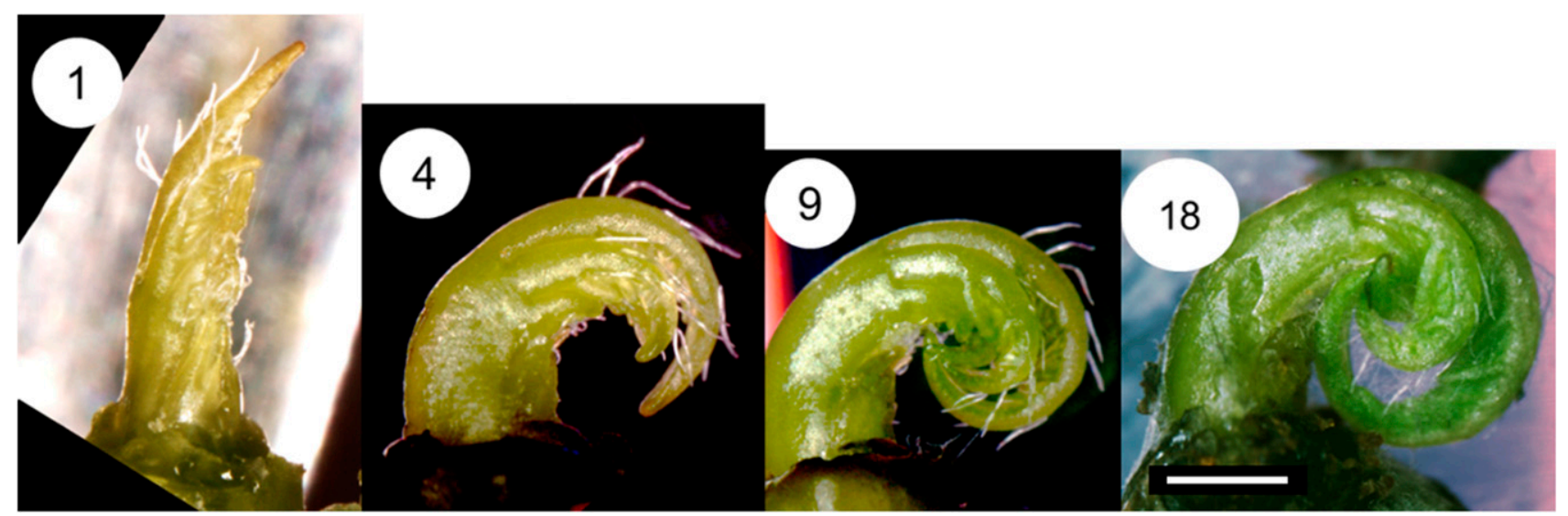

Fig. 4. Ablation of one leaf induces growth and curving of the opposite leaf. (A) Maple leaves form in pairs with a $90^{\circ}$ lateral shift in position. A pair of primordia (a's) develop inside the previous pair (b's). One leaf from the pair (b) was removed to reveal the younger pair (a) growing inside. (B) Longitudinal sections of young primordia. The cells in the abaxial side are much larger than ones on the adaxial side. (C, D) Shoot apices were dissected and then tissue cultured. Both leaves in a pair were left intact for the control samples (C), or one leaf was removed in the ablated samples (D). In the ablated samples, the remaining leaf grew into the space previously occupied by the ablated leaf. The leaf grew curving inward; within a leaf, each lobe curved independently. Numbers indicate days after surgery. Scale bars $=500 \mu \mathrm{m}$.

and grow at the same rate opposite to one another (Fig. 1A; Couturier et al., 2009). The lamina of mature leaf typically consists of three large lobes and numerous smaller lobes within a large lobe (Fig. 1B). The apex of each lobe corresponds to the tip of a vein. Lamina folding occurs along the veins (Fig. 1C), initiating around the time when small nicks are visible at the pri- mordia margin in the middle of two future lobes (Fig. 1D, E). First, the three folds appear along the primary veins, and later more folds form along the secondary veins. Furthermore, folding spreads in the proximal to distal direction. Therefore, a primordium contains folds and antifolds at varying stages of fold formation. 

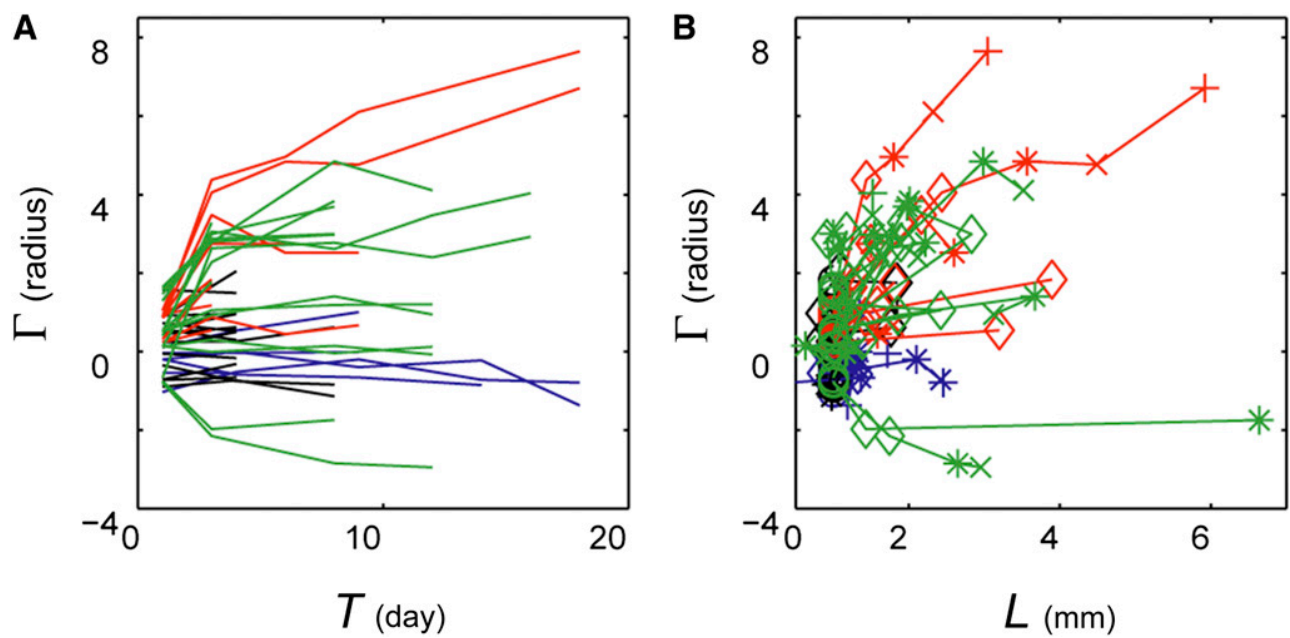

Fig. 5. Quantitative assessment of ablation induced growth and curving of the leaf. The total turning angle $\Gamma$ of the abaxial side of the midvein from the intact leaf along the central lobe (radian) as a function of (A) days after surgery $T$ or (B) length of the leaf $L$ (mm). Two biological replicates are indicated in red and green (for ablated samples) or black and blue (for nonablated controls). $\bigcirc, \diamond, *, \times$, and + denote value on 1, 3, 6, 9, 18 (red) and 1, 3, 8, 12,16 (green) days after surgery.

To gain more insights into what drives lamina folding, transverse sections of two primordia in the process of folding were examined. The areas of the lamina near the veins were observed at different stages of fold formation (Fig. 2A). The lamina on the abaxial side of the veins grows much more than the adaxial side. This hypergrowth seems to occur through more cell division and expansion. The differential growth may exert a compressive force on the adaxial side, since the epidermal cells on the adaxial side of the folds (the upper panel) and the abaxial side of the antifolds (the lower panel) are narrower toward the surface (Fig. 2C). Enhanced growth on the outer side of the leaf could impose folding on the lamina.

To quantify the correlation between the abaxial growth and the lamina folding, we measured the lamina thickness away from the veins $L$, the lamina thickness at the veins $V$, and the fold angle $\alpha$ (Fig. 2B). The fold angle was plotted as a function of the ratio $L / V$. When $L / V$ is close to 1 , which is when the abaxial hypergrowth has not happened yet, $\alpha$ is close to $180^{\circ}$, or the lamina is flat. When the ratio is small, $\alpha$ approaches $0^{\circ}$ (i.e., the fold is closed). Around the $L / V$ ratio of 0.4 , the fold does not close further but the abaxial side of the lamina along the veins continues to grow and form the rib. Overall, the bigger the rib becomes, the more folded the lamina $(r=-0.96, P<$ $10^{-4}, N=39$ ). Fold formation coincides with the corresponding vein's differential growth across the abaxial-adaxial axis. Interestingly, the rib cell differentiation that leads to the abaxial hypergrowth initiates before the onset of folding (Fig. 2Aa).

The dynamics of the antifold formation were also quantitatively examined from the same transverse sections. We measured the lamina thickness at vein $V$, which mostly reflects the abaxial growth, and the distance $A$ between the vein/fold and the nearest antifold (Fig. 3A, C). For each antifold, we plotted $\mathrm{A}$ as a function of $\mathrm{V}$. They were strongly linearly correlated $(r=$ $0.6846, P<\mathrm{e}^{-4}, N=42$ ). The distance between the vein and the antifold is directly proportional to the lamina thickness at the vein; in other words, the lamina extends proportionally to the rib enlargement. Next the relationship between the antifold formation and the vein thickness was examined. The antifold angle $\beta$ was plotted against the sum of the lamina thickness at two adjacent veins $V_{1}+V_{2}$ (Fig. 3B, D). There was a negative correlation between the two parameters $(r=-0.6862, P=0.0096$, $N=21$ ), indicating that the larger the abaxial side of the veins grows, the more folded the lamina becomes, leading to the antifolds.

Space-dependent growth of the primordia-We next examined the effects of physical constraints on kirigami leaf morphogenesis. The space available for a primordium's growth is determined by other primordia in the vicinity, which we removed by surgical ablation. Maple leaves emerge in decussate phyllotaxy inside the older leaves or bud scales (Fig. 4A). Young primordia are curved slightly inward, so that the lamina of the two opposite leaves meet each other in the middle. The curvature indicates that the abaxial side grows more than the adaxial side. Longitudinal sections show that the cells on the abaxial side begin to expand early in development (Fig. 4B). We dissected actively growing buds down to the youngest 3-4 pairs of primordia, cultured them on a solid medium, and observed the growth of the outermost primordia. We first verified that they could survive and grow in the cultured condition. Although fungal and bacterial infection consumed the plants in long-term incubations, we could observe leaf growth over several days to a few weeks.

One of the two pairing leaves was removed by surgical ablation, and the development of the remaining leaf was monitored. Of 25 samples total, two clearly unfolded within a day, indicating that physical constraint keeps folds in their place and is important for proper folding. The remaining 23 showed a common behavior in which the intact leaf grew extensively, especially on the abaxial side of the primordia, and curved into the space where the ablation took place (Fig. 4D, 5A, 5B). The increase in the total turning angle measured along the abaxial side of the central lobe in side view, is significantly correlated with the ratio between the final to initial length of the leaf ( $r=0.51, P=$ $0.01, N=23)$. There was no significant change in the control without the ablation $(r=-0.28, P=0.27, N=17$ ) (Fig. 4C, 5A, $5 \mathrm{~B})$. There was no correlation between the mean turning angle variation (mean taken along the vein) and the increase in leaf 

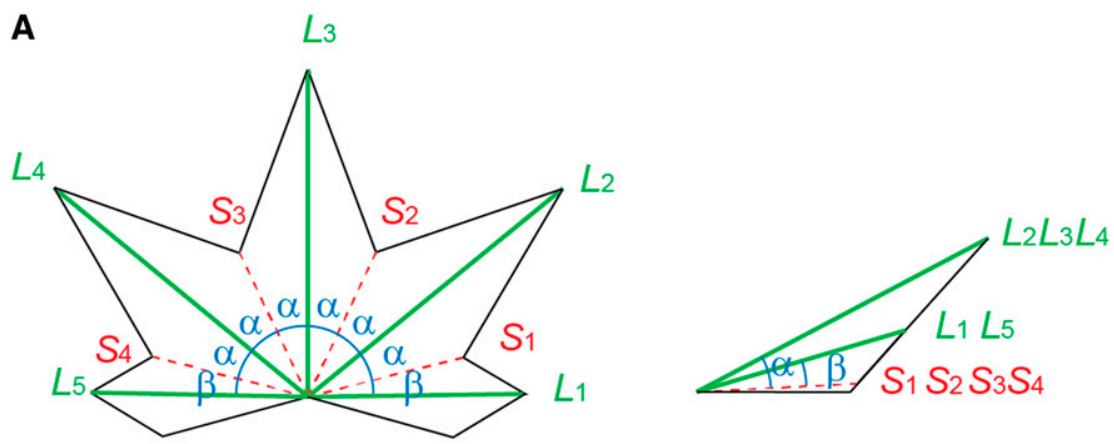

B
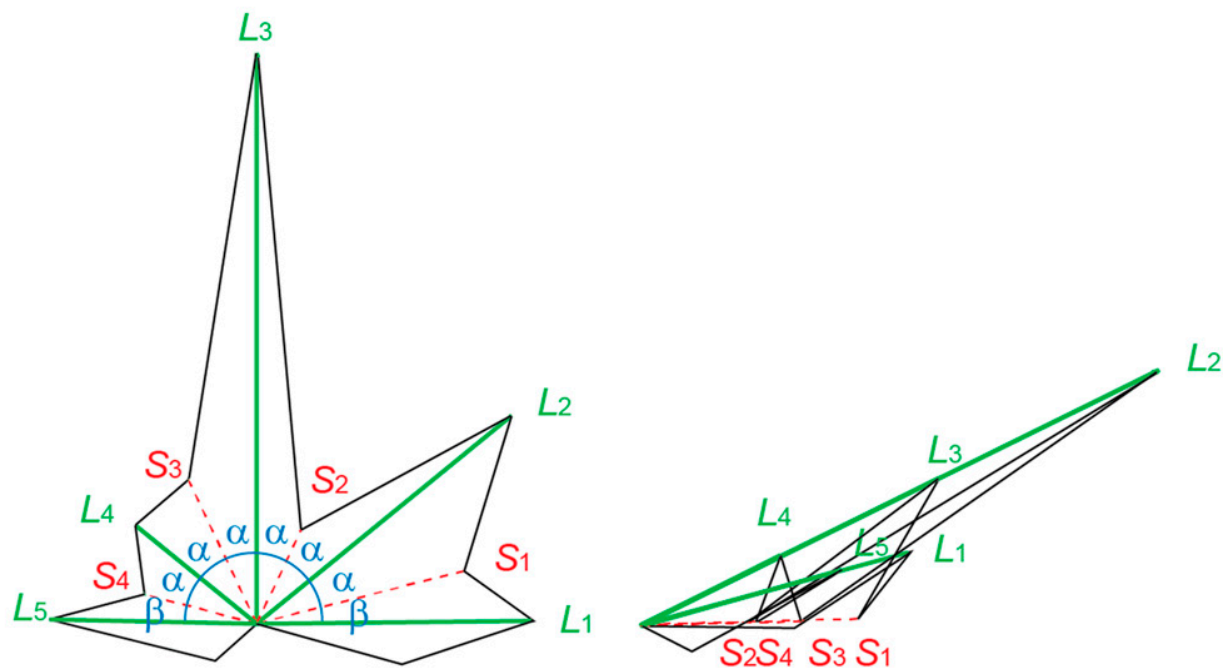

Fig. 6. Mathematical prediction of leaf morphology. Shapes of a hypothetical five-lobed leaf unfolded (left) and folded (right). The lobes/folds are marked green, the sinuses/antifolds red. (A) A leaf that respects the kirigami constraints and can be folded to align the lamina margin on a plane, as in naturally growing maple leaves. The most lateral lobes $L_{1}$ and $L_{5}$ have the shape of lamina characterized by $\alpha>\beta$. The heights of the lobes have the relationships: $L_{1}<L_{2}$, and $L_{5}<L_{4}$. The three central lobes $\left(L_{2}, L_{3}, L_{4}\right)$ have the same angle $\alpha$ with respect to the sinuses/antifolds $S_{1}, S_{2}, S_{3}, S_{4} ;$ so $L_{2}=L_{3}=L_{4}$, and $S_{1}=S_{2}=S_{3}=S_{4}$. (B) A leaf that does not respect the kirigami constraints and cannot be folded to align the lamina margin on a plane, as observed in the ablation experiment. The most lateral lobes $L_{1}$ and $L_{5}$ have the shape of lamina characterized by $\alpha>\beta$, but the heights of the lobes have the relationships: $L_{1}=L_{2}$, and $L_{5}>L_{4}$. The three central lobes $\left(L_{2}, L_{3}, L_{4}\right)$ hold the same angle $\alpha$ with respect to sinuses/antifolds $S_{1}, S_{2}, S_{3}, S_{4}$; however, it is not coherent with their respective lengths. For example, in this case, $L_{2}<L_{3}, L_{4}<L_{3}, S_{2}<S_{1}, S_{2}<S_{3}$, and $S_{4}<S_{3}$.

length $(\mathrm{r}=-0.01, p=0.94, \mathrm{~N}=23)$, indicating that the curvature was constant and that only the growing tip curves during the growth. The petiole of the leaf was already curved at the time of ablation and did not curve farther (Fig. 5B). When the leaf survived beyond $3 \mathrm{~d}$, all the lobes curved independently from one another (Fig. 4D).

Since technical difficulties prevented full maturation of the leaves in the cultured condition, we theoretically deduced the final morphology of the maple leaves growing with or without the physical constraints from the opposite leaf (Fig. 6). The number of lobes and sinuses and the angles in between them are fixed due to vein pattern. Each lobe could grow indefinitely and independently from the others. It has been observed for natural maple leaves, however, that the final sizes of the lobes and sinuses are not random, but follow numerical relations that make them foldable to align their margins on one plane (Couturier et al., 2009). If we remove the constraint of the opposite leaf, the lamina does not grow on a plane, as observed in the ablation assay. The resulting mature leaf will no longer hold these relationships among the shapes of the lobes and sinuses. The kirigami effects separate the successive lobes and sinuses at the same angle, making them also nearly equal in size (Fig. 6A).
A leaf grown without steric constraints would be different; the sizes of the lobes and sinuses could be more random (Fig. 6B). Moreover, morphology could vary leaf to leaf within an individual plant.

To gain more detailed information on the ablation effects, we examined longitudinal sections of the apices that were cultured for $3 \mathrm{~d}$ after the surgery (Fig. 7). In the control samples, in which the primordia were left inside the bud, one can draw an almost straight line between the two pairing primordia, between the ordered rows of lamina folds (Fig. 7A). When the leaves were cultured without the bud, the lamina folds lost some of the even and uniform organization (Fig. 7B). In ablated samples, the remaining leaf of the pair, as well as the younger primordia inside, grew into the newly available space (Fig. 7C-7E). The lamina of the remaining leaf lost its ordered pattern of folding and was unfolded in some places, being twisted almost $90^{\circ}$ and resembling transverse sections. Therefore, the physical constraints from the older primordia and the opposite primordium enforce the proper lamina folding. Interestingly, the excess growth still conformed to the space-filling rules (Couturier et al., 2011) and stopped when the lamina came close to the new boundary of physical constraints even at the wounded sites (Fig. 7D, 7E). 
A

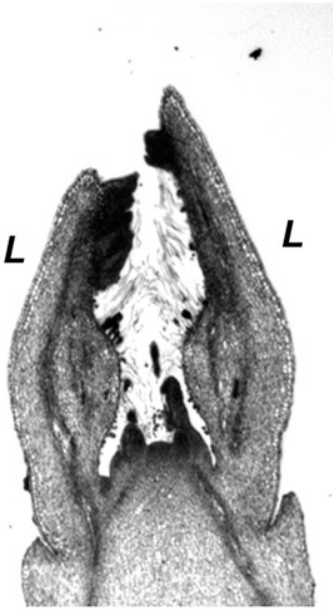

B

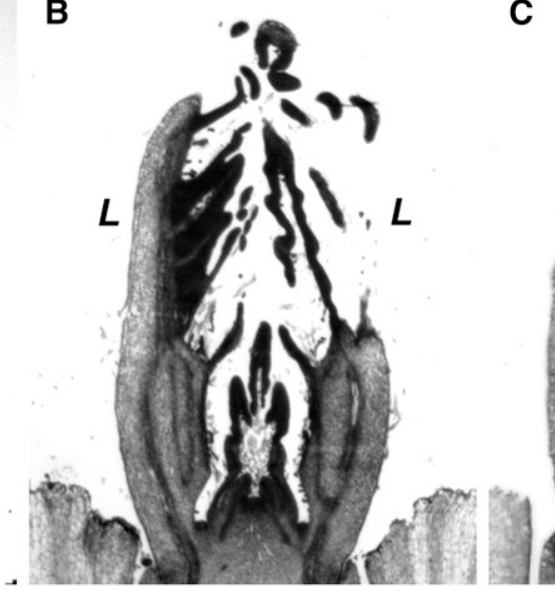

C

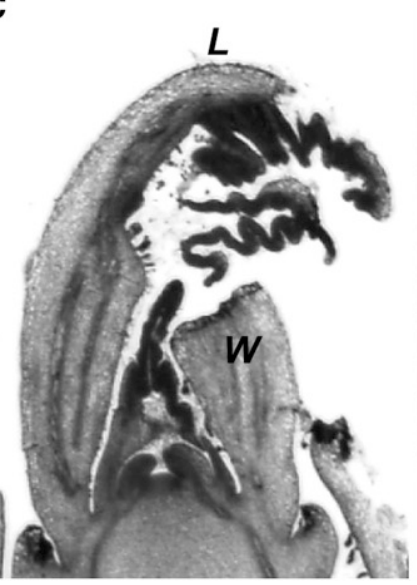

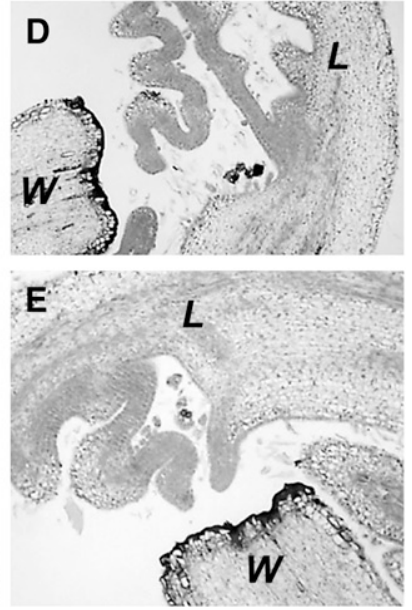

Fig. 7. Histological examination of the ablation effects. Longitudinal sections of tissue-cultured maple shoot apices. The apices were cultured for 3 days after surgery. (A) Representative of controls, in which leaves were left inside the bud. The apices were dissected down to 3-4 pairs of primordia before fixation. Opposite leaves meet each other in the middle, and the lamina $(L)$ is folded in a uniform and ordered manner. (B) Representative of another kind of controls in which the apices were dissected down to 3-4 pairs of primordia before culturing. The opposite leaves still meet each other in the middle, but the order of the folds is disturbed. (C) Representative of ablated samples in which one leaf was removed from a pair. Ablation resulted in induction of growth toward the ablated area. The lamina showed clearly abnormal folding. (D, E) Close-ups of the remaining leaf near the ablation wound. Space-dependent growth regulation is apparent even at the wound sites (marked $W$ ).

A priori this phenomenon could be a wound effect due to the surgery. To address this concern, we conducted a partial ablation experiment by removing only the central lobe from one of two pairing leaves, leaving all the lateral lobes (Fig. 8A). In this case, the central lobe of the intact leaf curved toward where ablation took place. The lateral lobes, which were still constrained by the lateral lobes of the opposite leaf, grew normally and did not curve $(N=4)$ (Fig. 8B). Even when the leaf did not grow much over several days, a clear curving occurred within the first day without any growth $(N=8)$ (Fig. $8 \mathrm{C})$, which may indicate the presence of residual stresses (i.e., the inhomogeneous growth of the different layers of the lamina creates stress inside, which was released upon the ablation). If curving were a wound effect, the whole leaf would have been affected, as well as the half-ablated leaf itself. Moreover, similar curving growth is found naturally, when the leaves grow outside of the bud and without physical constraints during their development (Fig. 8D). Therefore, ablation-induced leaf curving is a physiological response.

\section{DISCUSSION}

To uncover the biological mechanisms behind the kirigami regulation of palmate leaf shape, we observed how maple leaves fold and grow during their development. A flat tissue that extends more on one side than on the other is geometricaly equivalent to curving or folding the tissue toward the side of less expansion. We observed in cytological sections that this abaxial growth is concentrated behind the veins and that the asymmetric growth of the lamina along the veins and the fold formation are correlated. These correlations point to the possibility of coregulation; in other words, abaxial growth along the vein, lamina extension, and folding are all controlled by a common regulator. Alternatively, lamina growth may regulate folding or vice versa. The fact that folds are not positioned randomly but are always localized on the veins suggests dependency between the two phenomena. The rib cell differentiation that will drive the enhanced growth on the abaxial side of the vein begins prior to lamina folding (Fig. 2A). Therefore, the abaxial hypergrowth along the vein can be the driving force for fold formation and probably imposes folding on the lamina. As folds tighten, the lamina grows and creates the antifolds. The positions of the folds are specified by the pre-existing venation, whereas the positions of antifolds may be decided by the spontaneous folding of the extending lamina in the midline between the two folds as a topological consequence. The physical constraints from the other primordia restrict the space in which the lamina can extend and would facilitate antifold formation. Small nicks were observed on the lamina margin that mark the midpoint between two adjacent veins prior to folding (Fig. 1D, 1E); such incisions could demarcate antifold formation to start at that specific location.

Surgical ablation also indicated the importance of the enhanced growth on the abaxial side of the lamina in regulation of the space-dependent growth. Because of the excess abaxial growth in the proximo-distal direction, a primordium curves toward and meets the opposite leaf on the middle plane. Once free from the physical constraints imposed by the other leaves, a primordium quickly grew into the newly available space while maintaining its curvature (Figs. 4, 5), suggesting that physical constraints limit the growth at the both adaxial and abaxial sides of the lamina. The geometrical calculation predicts that without the growth inhibition from the opposite primordium, the lobes and sinuses would become less even in shape and size (Fig. 6).

The newly gained mechanistic information supplemented the original kirigami model (Couturier et al., 2009), which is now revised as follows (Fig. 9A). The enhanced growth on the abaxial side of the lamina along the veins causes lamina folding. The same enhanced growth on the abaxial side curves the primordia slightly inward so that the two opposing primordia meet each other at the plane in the middle. Proper folding also depends on the geometry of the space available for the new primordia to grow, which are defined by the older primordia in the vicinity 
A
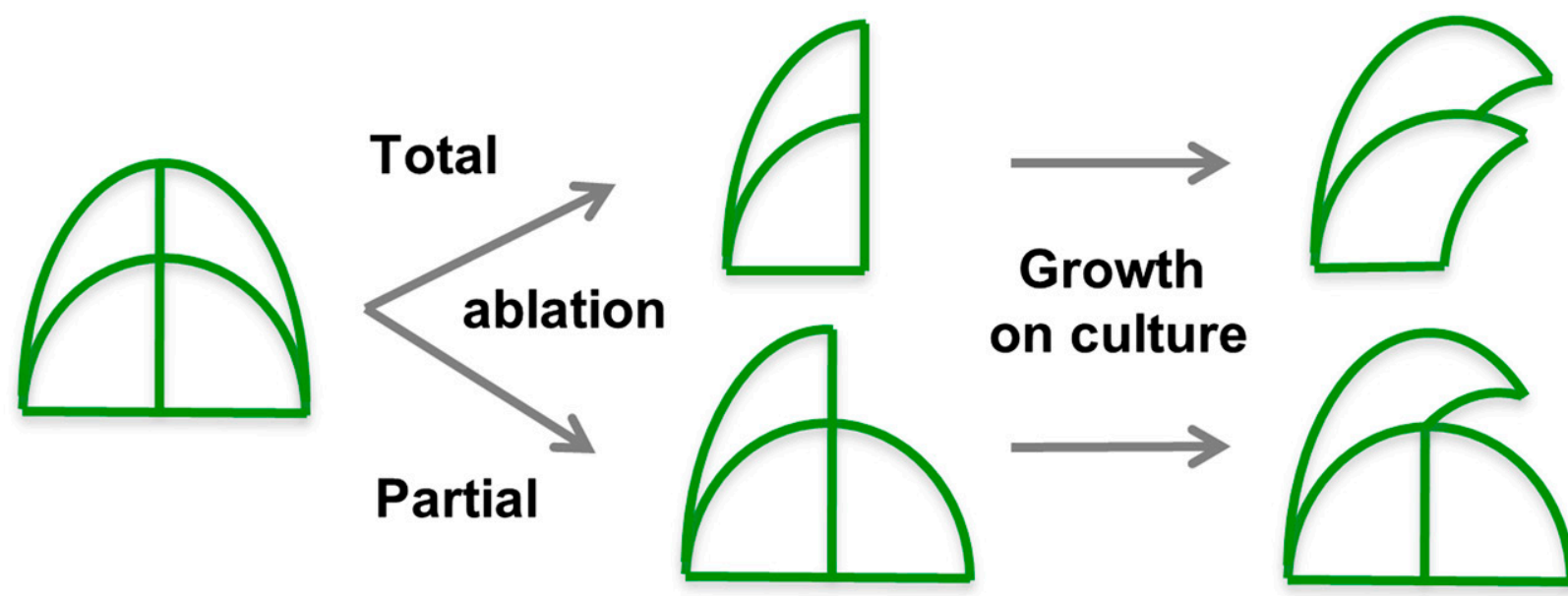

\section{on culture}
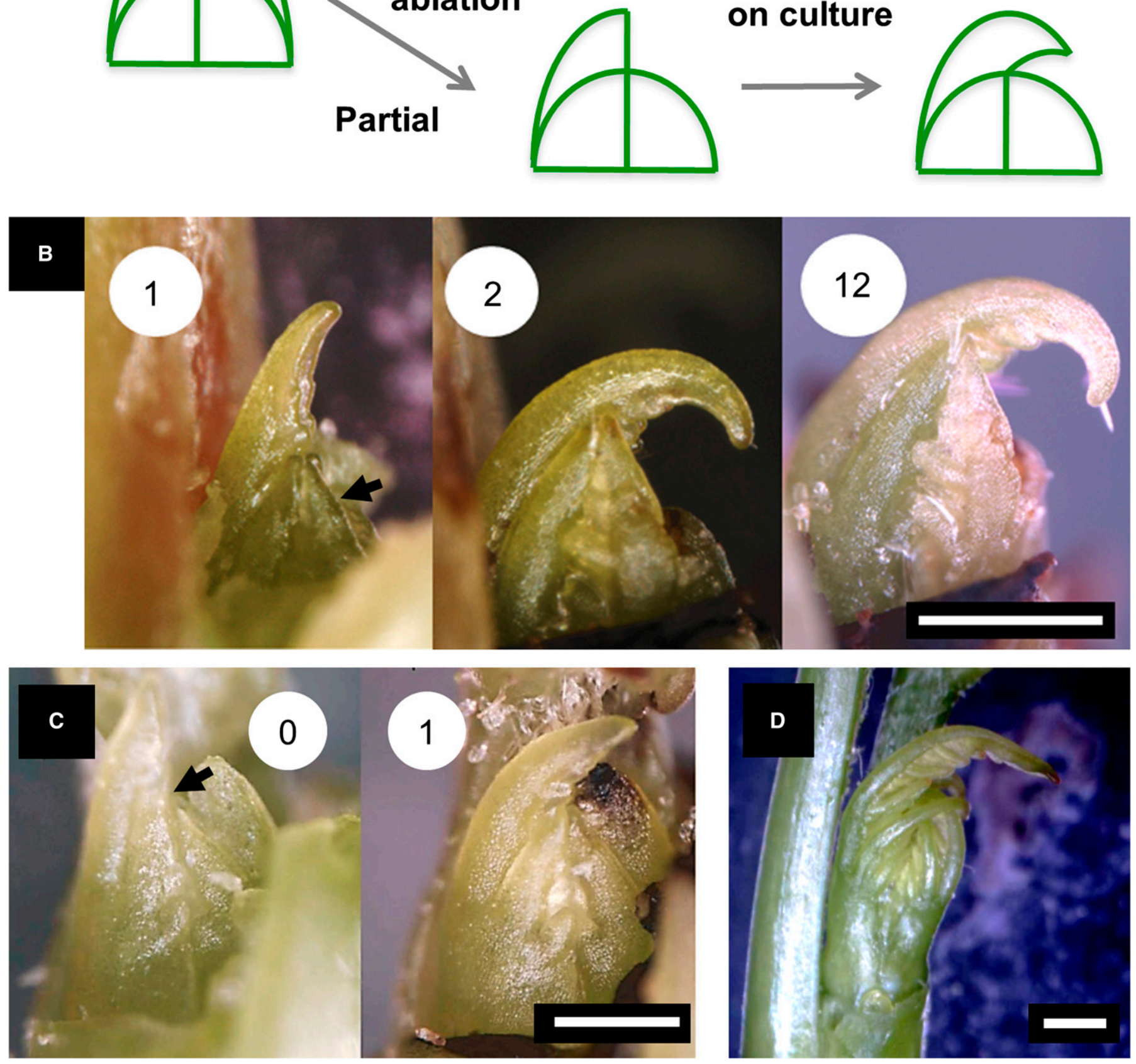

Fig. 8. Ablation affects growth locally. (A) Schematics of total and partial ablation. Either the entire leaf or only the central lobe was removed from a pair of facing leaves and tissue cultured for a day. Lobes curve into the newly available space independently. (B, C) The central lobe was removed from a leaf at two different heights, at lower (B) and higher (C) positions. The central lobe of the intact leaf grew and curved into the newly available space. The remaining parts prevented the curving of the corresponding lateral lobes of the intact leaf. Arrows point at the ablation sites. Numbers indicate day(s) after dissection. Scale bars $=500 \mu \mathrm{m}$. (D) Naturally occurring neo-morphed leaf of Acer pseudoplatanus, which grew outside the bud. Naturally free from surrounding physical constraints, lobes were loose, and the leaf curved without any ablation. Scale bar $=1 \mathrm{~mm}$. 
A

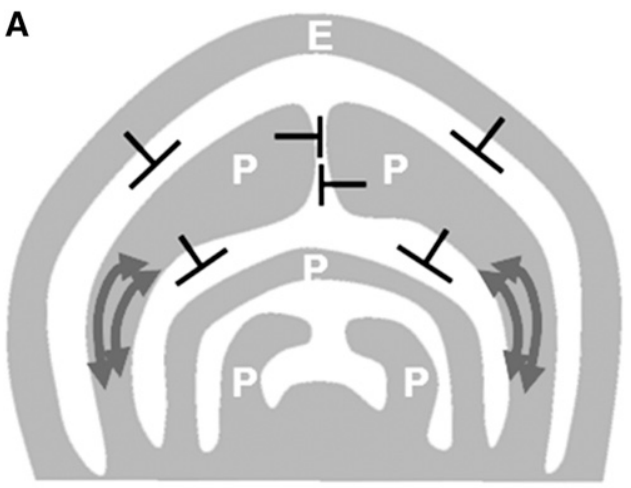

B

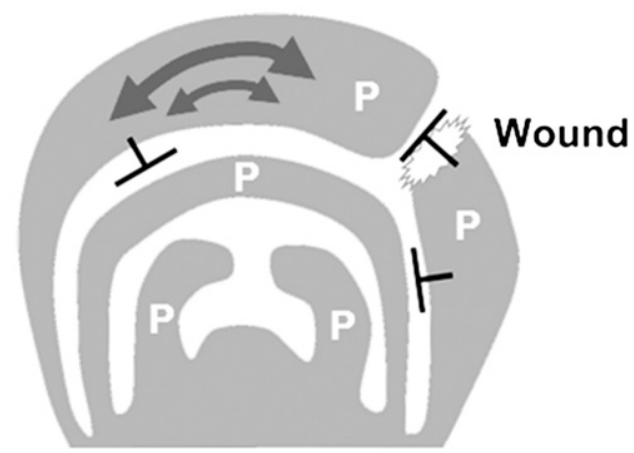

Fig. 9. Mechanism of kirigami leaf shape regulation via folding and physical constraints. (A, B) Summary of growth regulation by available space in normal conditions (A) or when one leaf was ablated from a pair (B). The envelope (designated E) made of the bud scales or older primordia, as well as the opposite leaf, set the physical constraint for lamina folding and growth. The lamina folds along primary and secondary veins inside the envelope, representing the paper-folding part of kirigami. The lamina grows until it fills the space, i.e., until they reach surrounding primordia. Because growth (double headed arrow) is enhanced on the abaxial side, the pairing leaves grow curving slightly inward, meeting one another in the middle (A). Facing leaves inhibit each other's growth, acting as the paper-cutting part of kirigami. When one leaf is removed, the remaining leaf grows excessively, while maintaining the curvature inward (B). The contact inhibition does not require normal surfaces on both ends, since it was also observed at the wound sites. P, primordia.

(Fig. 7). In normal apices, the division plane between two pairing primordia appears almost flat, but slightly fluctuates on close inspection (Fig. 1A), indicating reciprocal growth limitation all along the margins of the primodia. Two opposing leaves inhibit growth of each other and stabilize at the central plane by mutual contact, exerting the cutting effect of kirigami.

A common mechanism-abaxial hypergrowth along the vein -mediates the ways by which the leaves fold their lamina and determine the geometry of the space inside the bud and thus surfaced as the critical developmental process for the kirigami model of leaf morphogenesis. The fact that a common process underlies both folding and space-filling growth of the primordia could explain why folding and the kirigami property coincide in evolution (Couturier et al., 2009). Lamina folding along the veins in the primordial stages occurred many times independently during the angiosperm evolution (Couturier et al., 2009), indicating that this evolutionary transition probably does not require complicated genetic changes. The observations in the maple could apply generally to the other dicotyledonous species with kirigami leaves.

Surgical ablation also revealed some mechanistic clues on how the steric constraints inhibit growth of the primordia. It requires fine regulation that probably involves perception and response to physical contacts (Williams, 1975; Couturier et al., 2011). The fact that partial ablation could induce local growth indicates that spatial exploration takes place locally in each lobe and sinus, if not all over the lamina or primordia. Perhaps the simplest explanation would be to draw an analogy between growth of the lamina in the bud and expansion of a piece of gel confined inside a box. The gel absorbs water and increases its volume until it reaches the surface of the box. The pressure exerted by the wall of the box stops the flux of water into the gel. It is a passive, spontaneous phenomenon and can occur when the force exerted by growth is smaller than that from the physical barrier.

Alternatively, growing primordia may actively recognize physical contact with chemical or physical signals. A primordium could sense physical constraints when it is exposed to a chemical signal present on the other primordia. Interestingly, the maple leaf primordia do not seem to require mutual communication, since primordia showed contact-inhibited growth even at the wound sites that lack a normal surface (Figs. 7D, 7E, 9B). The contact regulation could also be mediated by mechanical stimuli. Touch stimuli and physical stresses are known to influence plant growth via modulation of gene activities (Braam, 2005; Coutand and Moulia, 2000). Repetitive touch application inhibits growth throughout the plant body (Braam, 2005), while a site-specific application of loads induced a more local response (Coutand and Moulia, 2000). What we observed in the maple leaf primordia resembles the latter, since growth could be inhibited even at the suborgan levels (Fig. 8).

The kirigami model of palmate leaf development entails both molecular and nonmolecular regulation of morphogenesis. While steric constraints oversee the size and the shapes, the numbers and the positions of the lobes are determined by venation, which is patterned by the dynamic flow of the phytohormone auxin (Nelson and Dengler, 1997; Sieburth, 1999; Scarpella et al., 2006). The initiation of a lobe or leaflet on a primordium starts with local accumulation of auxin, just like leaf initiation (Reinhardt et al., 2000; Nikovics et al., 2006; Barkoulas et al., 2008; Blein et al., 2008; Koenig et al., 2009; Kawamura et al., 2010). It promotes outgrowth, and $C U C$ genes are expressed on the surrounding area to mark the border of the outgrowth. Since the kirigami leaves develop through repetitive reiteration of outgrowth (lobe) and border (sinus), it would be interesting to see if folds and antifolds can induce or maintain auxin accumulation and $C U C$ gene expression, respectively. Physical forces also influence the direction of cell growth and division, probably through regulation of the microtubule organization (Lintilhac and Vesecky, 1984; Hamant et al., 2008). We anticipate that future investigations of kirigami leaf morphogenesis will address the integration of chemical and physical regulation of biological form.

\section{LITERATURE CITED}

Azri, W., C. Chambon, S. Herbette, N. Brunel, C. Coutand, J.-C. Leplé, I. Ben Rejeb, et AL. 2009. Proteome analysis of apical and basal regions of poplar stems under gravitropic stimulation. Physiologia Plantarum 136: 193-208.

Barkoulas, M., A. Hay, E. Kougioumoutzi, and M. Tsiantis. 2008. A developmental framework for dissected leaf formation in the Arabidospis relative Cardamine hirsuta. Nature Genetics 40: 1136-1141. 
Blein, T., A. Pulido, A. Vialette-Guiraud, K. Nikovics, H. Morin, A. Hay, I. E. JohansEn, ET AL. 2008. A conserved molecular framework for compound leaf development. Science 322: 1835-1839.

Braam, J. 2005. In touch: Plant responses to mechanical stimuli. New Phytologist 165: 373-389.

Braybrook, S. A., ANd C. Kuhlemeier. 2010. How a plant builds leaves. Plant Cell 22: 1006-1018.

Coutand, C., AND B. Moulia. 2000. Biomechanical study of the effect of a controlled bending on tomato stem elongation: Local strain sensing and spatial integration of the signal. Journal of Experimental Botany 51: $1825-1842$.

Couturier, E., S. Courrech du Pont, and S. Douady. 2009. A global regulation inducing the shape of growing folded leaves. PLOS ONE 4: e7968.

Couturier, E., S. Courrech du Pont, and S. Douady. 2011. The filling law: A general framework for leaf shape diversity and its consequences on folded leaves. Journal of Theoretical Biology 289: 47-64.

EFroni, I., Y. Eshed, ANd E. Lifschitz. 2010. Morphogenesis of simple and compound leaves: A critical review. The Plant Cell 22: 1019-1032.

Fleming, A. J. 2005. The control of leaf development. New Phytologist 166: 9-20.

Hamant, O., M. G. Heisler, H. Jönsson, P. Krupinsky, M. Uytteval, P. BoKov, F. Corson, ET AL. 2008. Developmental patterning by mechanical signals in Arabidopsis. Science 322: 1650-1655.

Hasson, A., T. Blein, AND P. LAUfs. 2010. Leaving the meristem behind: The genetic and molecular control of leaf patterning and morphogenesis. Comptes Rendus Biologies 333: 350-360.

Kawamura, E., G. Horiguchi, and H. Tsukaya. 2010. Mechanisms of leaf tooth formation in Arabidopsis. Plant Journal 62: 429-441.
Koenig, D., E. Bayer, J. Kang, C. Kuhlemeier, and N. Sinha. 2009. Auxin patterns Solanum lycopersicum leaf morphogenesis. Development 136: 2997-3006.

Lintilhac, P. M., And T. B. Vesecky. 1984. Stress-induced alignment of division plane in plant tissues grown in vitro. Nature 307: 363-364.

Moon, J., And S. Hake. 2010. How a leaf gets its shape. Current Opinion in Plant Biology 14: 1-7.

Nelson, T., And N. Dengler. 1997. Leaf vascular pattern formation. Plant Cell 9: 1121-1135.

Nikovics, K., T. Blein, A. Peaucelle, T. Ishida, H. Morin, M. Aida, AND P. Laufs. 2006. The balance between the miR164A and CUC2 genes controls leaf margin serration in Arabidopsis. Plant Cell 18: 2929-2945.

Prusinkiewicz, P., and P. Barbier de Reuille. 2010. Constraints of space in plant development. Journal of Experimental Botany 61: 2117-2129.

Reinhardt, D., T. Mandel, and C. Kuhlemeier. 2000. Auxin regulates the initiation and radial position of plant lateral organs. Plant Cell 12: 507-518.

Scarpella, E., D. Marcos, J. Friml, and T. Berleth. 2006. Control of leaf vascular patterning by polar auxin transport. Genes \& Development 20: 1015-1027.

Sieburth, L. E. 1999. Auxin is required for leaf vein pattern in Arabidopsis. Plant Physiology 121: 1179-1190.

SinHA, N. 1999. Leaf development in angiosperms. Annual Review of Plant Physiology and Plant Molecular Biology 50: 419-446.

Williams, R. F. 1975. The shoot apex and leaf growth. Cambridge University Press, New York, New York, USA. 